\title{
Minerva and Virgil - a program (Notes on Karpathy et al. 2014)
}

\author{
Camille Akmut
}

A proof of concept.

\begin{abstract}
Recent advances in machine learning and computer vision have resulted in the accurate and consistent identification of objects in images and videos. In this paper, a program named "Minerva and Virgil" is described whose purpose it is to counter State brutality, a term that we propose as the generalization of the already established "police brutality", by making use of these technologies.
\end{abstract}

\section{Introduction}

The philosophers of the Industrial age, of which Bentham had distinguished himself as the most industrious discipline and bondage philosopher of all, had given much thought to the issue of the control of the "masses" (by which was meant the working poor, whose habits and ways, and needs of freedom, rights and justice, were cause of much discontentment to the elites, as they are today).

Historians of the Middle ages have pointed out to us that this was by then in fact nothing new, the aggregation of populations around certain cities and areas had emerged as a phenomenon already in the Early Middle ages. However, this feature which prefigured our modern lives grew exponentially afterwards.

One solution that emerged then was the Panopticon, a system with which, from a single vantage point, all inmates of a prison could be observed. 
But, such notions rested, and could only rest on the assumption that these solutions were the privilege of the few, imposed on the many : a Panopticon would have required land and a budget that made it mostly the prerogative of the State.

New techniques, relying on the combination of machine learning and artificial intelligence with computer graphics, the combination of which is a subfield called computer vision, also visual recognition, have emerged in the form of software that can reliably identify objects from an image or video.

Most current efforts have concentrated on making commercial or policing uses of these technologies.

But, what the administrators and philosophers of this age had not anticipated, was that, because these new means of discipline and surveillance had taken on the form of the immaterial, the main obstacle that prevented previous generations from reversing their uses, primarily the capital needed, pointed out above (stones, land, labor, etc.), was suddenly lifted.

\subsection{About the name}

Minerva : The goddess Minerva has many faces : Minerva of Wisdom here, Minerva of Peace there, Minerva of War with others yet. Her meaning and uses changed as did the society that worshiped, and had made her.

Virgil : In Dante's Divine Comedy, Virgil, "poet of poets" and one of the most widely respected and admired figures in all of Roman culture and history, can be a guide to Dante, but only up to a certain point, after which Dante must continue his journey alone.

Because much of computer science culture tends toward the shortening of all things, such a program's alias could be MiV (Minerva in one way, Virgil in the other), MinVir (reminiscent of the MinMax algorithm), Maeve or any of its variations (e.g. Maiv, Maev). ${ }^{1}$

\section{Theoretical background}

\section{1 "AI", "ML", "deep learning", and neural networks}

In contemporary computer science, artificial intelligence ("AI") and machine learning (shortened "ML") are the two broad categories that deal with the issue of how to make intelligent systems : software that can read, talk, write, and see, and ultimately think.

To help make sense of the multitude of designations, we follow Chollet who presents artificial intelligence as the broadest category, machine learning a part (or subset) of it, and deep learning in turn a part (or subset, subfield, branch) of machine learning. This presentation is compatible and overlaps with that of

\footnotetext{
${ }^{1}$ Alternatively, each could represent a different implementation.
} 
Goodfellow et al. ${ }^{2} .^{3}$

One approach that has proved successful in the classification of images ${ }^{4}$ and videos $^{5}$, and the intelligent analysis of visual material in general ${ }^{67}$, is called "deep learning", a contemporary terminology strongly associated, if not synonymous, with the other, "neural networks" 8 (or simply "neural nets"9).

A definition of deep learning was given by Goodfellow et al. as follows : "deep learning (...) is an approach to AI. Specifically, it is a type of machine learning, a technique that enables computers to improve with experience and data." 10

In giving their definition, the authors go much further, they add : "We contend that machine learning is the only viable approach to building AI systems that can operate in complicated real-world environments." (as opposed to the constrained, and, in that sense, more simple worlds of systems such as chess, which they mention in previous developments). ${ }^{11}$

\subsection{Visual recognition}

A good entry point into the topic of visual recognition, and specifically visual recognition applied to videos, is Karpathy et al. 2014, "Large-scale Video Classification with Convolutional Neural Networks" ${ }^{12}$.

Earlier research into the problem of visual recognition appears to have been mostly done on images ${ }^{13}$.

Although, these two problems are not fundamentally different if considering that videos are constituted by many, successive images.

\footnotetext{
${ }^{2}$ Chollet 2017, Goodfellow et al. 2016

${ }^{3}$ If one were to draw out their presentations, would "neural networks" be subsumed under deep learning? As always, it is likely that such classifications are the product of fights and infights with tenors of the discipline, and disciplines, but this is out of the scope of this treatment.

${ }^{4}$ Le Cun et al. 1989, Lecun et al. 1998.

${ }^{5}$ Karpathy et al. 2014.

${ }^{6}$ We mean any kind or sort of - in the broadest sense - moving images. See the next note for examples of applications that fall outside of the expected.

${ }^{7}$ In SethBling 2015 and 2015b (the latter for the limitations of the chosen method) and the separate 2017, we find applications of machine learning and neural networks to the specific problem of video games (and specifically video games navigation, from the perspective of a traditionally human player, by a non-human one). See also Stanley and Miikkulainen 2002.

${ }^{8} \mathrm{~A}$ much needed and essential history of these varying appellations, and their changing and waning popularity and uses over time, is provided in "1.2 Historical Trends in Deep Learning" of Goodfellow et al. 2016. A historical perspective is also present in Chollet 2017, specifically chapter 1 and section 1.2 .

${ }^{9}$ Chollet $2017: 15$.

${ }^{10}$ Goodfellow et al. $2016: 8$.

${ }^{11}$ Ibid.

${ }^{12}$ The paper website https://cs.stanford.edu/people/karpathy/deepvideo/ offers many additional resources, in addition to the article alone. Although, the paper itself goes into detail, and explains the methods they have used and the data they have relied on, and some of the problems they have faced.

${ }^{13} \mathrm{~A}$ good entry point here are the two papers by LeCun et al..
} 
(A video is a succession of images ${ }^{14}$, an image is a combination of many pixels, while pixels are a unit that each hold a combination ${ }^{15}$ of number values that together determine color ${ }^{16}$ and position in the 2 -dimensional plane, $\mathrm{x}$-axis and y-axis coordinates.)

Nonetheless, videos present particular challenges due to size and length and labeling as well as other issues explained by Karpathy et al..

This research stands out both due to their results ${ }^{17}$, and the amount of training data that they were able to put together for the purposes of visual recognition applied to video material specifically.

Because, they have based their approach on "convolutional neural networks", we follow their approach.

\subsection{Convolutional Neural Networks}

Convolutional neural networks, a special class or type of neural networks, represent one way to obtain the desired result i.e. the consistent correct identification of an object (or objects) from a video.

In their abstract, Karpathy et al. state unambiguously : "Convolutional Neural Networks (...) have been established as a powerful class of models for image recognition problems." 18

Convolutional neural networks are found abbreviated in the literature and elsewhere as "CNN", also "convnets" 19 , or just "convolutional networks" 20.

Where the topic of convolutional neural networks is concerned, a majority of researchers ${ }^{21}$ refer to the research done by LeCun and his collaborators, in particular LeCun et al. 1998, "Gradient-Based Learning Applied to Document Recognition" ${ }^{22}$, and with less frequency Le Cun et al. 1989, "Handwritten digit recognition with a back-propagation network" ${ }^{23}$.

As such, it would seem appropriate to cite from him where a definition for convolutional neural networks is sought. "Convolutional Neural Networks are a special kind of multi-layer neural networks. Like almost every other neural networks they are trained with a version of the back-propagation algorithm. Where they differ is in the architecture. Convolutional Neural Networks are

\footnotetext{
${ }^{14}$ In the range of about 10 to 60 per second depending upon video quality (varying from old surveillance installations to recent consumer including phone recording technology).

${ }^{15}$ Even though we use the term combination here we really mean the mathematical concept of a matrix. In the literature regarding machine learning, the concept of tensor is often used instead : whereby a matrix is considered to be a specific case of a tensor, and whereby specific cases of tensors are assigned ranks (while from the point of view of matrices, tensors would constitute specific cases of matrices in higher dimensions, as vectors in lower, but we leave this issue here).

${ }^{16}$ E.g. RGB color model.

${ }^{17}$ https://cs.stanford.edu/people/karpathy/deepvideo/cnn_video_classify_demo.m4v

${ }^{18}$ This faith in convolutional neural networks is echoed in other publications (for example Chollet 2017).

${ }^{19}$ Chollet 2017 consistently uses this abbreviation / terminology / designation.

${ }^{20}$ Bishop 2006, Goodfellow et al. 2016.

${ }^{21}$ This applies to most, if not all of the authors consulted and mentioned here.

${ }^{22}$ At the time of writing, this publication has over 15,000 citations.

${ }^{23}$ As of now, a little over 2,000 citations.
} 
designed to recognize visual patterns directly from pixel images with minimal preprocessing." 24

But, going a step backwards, the older of the two writings is of interest (earlier publications have the advantage that they necessitate less accumulated knowledge of the field, which can often represent a seemingly insurmountable barrier to entry for new and late entrants ${ }^{2526}$ ).

Providing a - as always much needed - historical context for this publication, Chollet writes: "The first successful practical application of neural nets came in 1989 from Bell Labs, when Yann LeCun combined the earlier ideas of convolutional neural networks and backpropagation, and applied them to the problem of classifying handwritten digits. The resulting network, dubbed LeNet, was used by the United States Postal Service in the 1990s to automate the reading of ZIP codes on mail envelopes." 2728

An authoritative treatment of machine learning is given in The Elements of Statistical Learning by Hastie et al. ${ }^{29}$, whose chapter 11 is on neural networks ${ }^{30}$.

Table $11.1^{31}$ gives an overview of the performance of different neural networks applied to the same dataset. On that same page, convolutional networks are mentioned for the first time, and through that table we learn that the best performing neural networks ( $>90 \%$ correct guesses) belong to that class ${ }^{32}$. (This is all based on the U.S. Postal Service dataset of handwritten ZIP codes and the results found in Le Cun et al. 1989 mentioned above.)

\footnotetext{
24 "LeNet-5, convolutional neural networks", http://yann.lecun.com/exdb/lenet/.

${ }^{25}$ Students, self-learners, graduates from other disciplines, or even researchers from other, disconnected subfields.

${ }^{26}$ This advantage however only exists if the author has survived or is thought likely to survive the test of time. In The Structures of Scientific Revolutions, Kuhn has already written much of what can be said about survival cycles, and the interaction of professional, social and biological death in the sciences (a topic of particular significance to this field).

${ }^{27}$ Chollet 2017 : 15.

${ }^{28}$ The value of such developments cannot be overstated, as they - as they do in mathematics where there are referred to as "motivations" (a term that can be understood in both a neutral and non-neutral sense) - help make sense of why the current approaches should be preferred instead, otherwise leaving the user and student or reader in some sort of confused and arbitrary state of not knowing why, but only how. In general, it must be posited that much is wrong with how education is conceived and conducted in both of these disciplines, although newer approaches to teaching them, combined with new forms of science popularization and the specific subfield of mathematics education try desperately to address and are moderately successful in tackling these problems. (While the high-from-up-above-the-clouds, idealistic developments of this generation's impenetrable Hegelian, I. H., provide little to no insights, except into the mechanisms of intellectual stardom. In later publications, if at all, he will only be referred to as Feuerbach of the sciences.)

${ }^{29} \mathrm{~A}$ more approachable presentation of the material present in this book is given by the same authors in An Introduction to Statistical Learning.

${ }^{30}$ In this never-ending maze of superficially exclusive but mostly compatible appellations, which result from and reflects the great variety of disciplines that computer science in general draws from, and artificial intelligence and machine learning specifically, perhaps more so than any other of its branches, statistics, psychology, medicine, philosophy, logic, mathematics, this reference book provides another example of this great diversity, which can also mean great confusion, where machine learning here is called "statistical learning".

${ }^{31} \mathrm{We}$ use the second edition referenced in the bibliography.

${ }^{32}$ Hastie et al. $2017: 407$.
} 
Of convolutional neural networks, and their limitations in certain forms, Bishop notes : "Given a sufficiently large training set, such a network could in principle yield a good solution to this problem and would learn the appropriate invariances by example. However, this approach ignores a key property of images, which is that nearby pixels are more strongly correlated than more distant pixels. Many of the modern approaches to computer vision exploit this property by extracting local features that depend only on small subregions of the image." 33

More in-depth and specialized treatments ${ }^{34}$ of convolutional neural networks are found in textbooks and lectures on "deep learning" 35 , "computer vision", "visual recognition" 36 , or such that contain "neural networks", in title or subtitle; while "pattern recognition" 37 and variants on "pattern" or "patterns", and similar appellations, seem to belong to a previous, now forgone era, and possibly minority schools.

To summarize : convolutional neural networks appear to be the current golden standard where image and video classification tasks are concerned. In ending the historical retrospective provided in his 2017 textbook, Chollet writes : "Since 2012, deep convolutional neural networks (...) have become the goto algorithm for all computer vision tasks; more generally, they work on all perceptual tasks." - echoing much of the same sentiments found in Karpathy et al..

\subsection{Gradient descent}

In "3.3. Learning", Karpathy et al. mention gradient descent.

\footnotetext{
${ }^{33}$ Bishop 2006 : 267.

${ }^{34}$ We hesitate to make every such statements be preceded by the mention of "current" or "currently", even though they really should be. To convince yourself of this fact, read the historical review present in Goodfellow et al. 2016, which is perhaps the most precious resource cited in this paper, along with the original article by Karpathy et al. : a mastery of the convoluted and confusing terminology of this field, alone, is a field of study in itself and in its own way. It should also probably be kept in mind that a consensus seems far from having been achieved in this field, with multiple schools, each with different historical origins and traditions, each with their own historically accumulated quirks and perks, adding to the general confusion. Not only this, but some authors, under the guise of teaching general courses of these subjects, or writing textbooks, of which it is assumed they offer a general view, are quick to bury certain approaches in 2 or 3 lines, while giving undue attention to others their own. Chollet offers some insight into these trends when he writes that "[a]round 2010, (...) neural networks were almost completely shunned by the scientific community at large" (Chollet 2016 : 17). As one can tell, unity in this field is far from having been achieved.

${ }^{35}$ Chapter 9 of Goodfellow et al. 2016 is entirely dedicated to convolutional networks, chapter 5 of Chollet 2017, "Deep learning for computer vision", focuses mostly on convolutional neural networks, chapter 13 of Geron 2017 is also entirely on convolutional neural networks, while the following chapter 14 is about "Reccurrent Neural Networks".

${ }^{36}$ Such is the case of the Stanford course with nomenclature CS231n, entitled "Convolutional Neural Networks for Visual Recognition", to which some of the original authors of the Karpathy et al. 2014 paper are attached. Videos of the Spring 2017 lectures have been made freely available online by Stanford.

${ }^{37}$ Chapter 5 of Bishop 2006 is on neural networks, with 5.5 .6 on convolutional networks specifically.
} 
Gradient is a term from mathematics, and specifically from calculus in multiple variables (multivariable calculus, vector calculus, possibly analysis, etc.). It is connected to the notion of a derivative of a function ${ }^{38}$. "The derivative $f^{\prime}\left(x_{0}\right)$ [of the function $f\left(x_{0}\right)$ ] measures the slope of the line tangent to the graph of $f$ at the point $\left(x_{0}, f\left(x_{0}\right)\right) . " 39$

It is used to describe the generalization of the derivative to several variables.

Gradient descent is an (optimization) algorithm. In providing a definition for convolutional neural networks, LeCun had mentioned backpropagation : gradient descent makes use of both backpropagation and the gradient.

Implementations of gradient descent in popular programming languages are available online $\mathrm{e}^{40}$. However, such algorithms are likely to be already implemented and available in (e.g. machine learning and deep learning) libraries, from which they can conveniently be called (e.g. function(param_1, .., param_n)) or used $^{41}$. This is true of most of the algorithms discussed here.

Specifically, Karpathy et al. mention using "Downpour Stochastic Gradient Descent", to optimize their models, of which Ruder writes: "Downpour SGD is an asynchronous variant of SGD that was used by Dean et al. in their DistBelief framework (predecessor to TensorFlow)" ${ }^{42}$. Both reference Dean et al. 2012, in which the algorithms appear in the appendix (in pseudo-code).

As such, it would make sense for a visual recognition application to make use of the aforementioned library, or one with equivalent functionality i.e. one that would make easy the use of the algorithms discussed here and previously ${ }^{43}$.

It is likely that since the publication of Karpathy et al.'s research, advances have been made, to which Ruder in his review article points to ${ }^{44}$.

\section{$3 \quad$ Testing and learning}

One of the conditions for convolutional neural networks to perform visual recognition tasks is the presence of training data, or multiple such databases (which can presumably either be treated as subsets of the same or possibly some form of test or control), with the general recommendation that the larger the number of (correctly formatted) items contained in them, the better : Geron cites insufficient quantity of training data as one of the main challenges of machine

\footnotetext{
${ }^{38}$ The derivative and, later, gradient are treated in chapter 2, "Differentiation in Several Variables", of Susan Colley's Vector Calculus.

${ }^{39}$ Ibid., "Some Preliminary Notation". The accompanying graphs, not rendered here, are always helpful in understanding such developments.

${ }^{40}$ https://en.wikipedia.org/wiki/Gradient_descent.

${ }^{41}$ Some functions may not be designed to be called directly but other functions that are will use them.

${ }^{42}$ Ruder 2016.

${ }^{43}$ Goodfellow et al. 2016 regularly include discussions of implementations, and note for instance, where convolution is concerned, that : "Many machine learning libraries implement cross-correlation but call it convolution." (where "cross-correlation (...) is the same as convolution but without flipping the kernel").

${ }^{44}$ Ruder 2016.
} 
learning ${ }^{45}$.

It is unclear if a threshold, or a minimal number, in this case of videos, exists or if researchers have agreed on terms of what makes a good collection of data for machine learning purposes, in particular when applied to visual recognition tasks and even more specifically to video material ${ }^{46}$. (Perhaps, the answer here is that "the proof of the pudding is in the eating". $)^{47}$

\subsection{Dataset}

One of the issues mentioned by Karpathy et al. was that, contrary to images, it had been hard to find or put together a large dataset of videos. In their case, their dataset, called Sports-1M, consists of 1 million such videos ${ }^{48}$.

A dataset for a program such as the one described would consist of footage of police officers, recorded both as they perform their normal duties and as they engage in brutality (and accordingly labeled).

A Police-1 $M$-like dataset remains to be created, and is not available in any practical form (i.e. one that would enable and encourage research, and a program to perform automatic operations on it) at the moment, as far as we know. Alternative projects and proposals could be for a Police-50K or PoliceBrutality$10 K$ dataset.

One of the problems would be that, contrary to sports, such a dataset would likely have to be segmented into single countries : kayaking is in principle the same everywhere, but police vary greatly from country to country, different laws, wildly different gun laws, different uniforms, etc., leading to different possible outcomes and situations; making, we believe, such a project only practical when limited to a single country to begin with (though such projects can run in parallel or separately, and should likely be done so).

This paper uses the term police, for which the Oxford Thesaurus gives the following list of synonyms :

police $n$.

1 constabulary, policemen, policewomen, police officers,

Colloq boys in blue, the (long arm of the) law, the cops, the gendarmes,

Slang the coppers, the fuzz, Brit the (Old) Bill, US the heat:

${ }^{46}$ Geron gives a number of pointers as to what pitfalls to watch out for when forming and structuring the data (Ibid., chapter 1).

${ }^{47} \mathrm{An}$ additional requirement is the necessary computation power, though this can likely be seen as a secondary concern for all sorts of reasons (due in part to the emergence of such things as "borrowed" computing power, variously called "cloud" and by other names.). Discussions surrounding the cost of OpenAI Five were split, but Karpathy et al. stress difficulties.

48 "From a practical standpoint, there are currently no video classification benchmarks that match the scale and variety of existing image datasets because videos are significantly more difficult to collect, annotate and store. To obtain sufficient amount of data needed to train our CNN architectures, we collected a new Sports-1M dataset, which consists of 1 million YouTube videos belonging to a taxonomy of 487 classes of sports."
} 
There was a bit of an argument, so we called the police.

They are also referred to in vernacular by other names.

The building of a dataset should account for all these terms, and their varying, culturally determined and historically situated uses (e.g. "beat pounder" is unlikely to bring any results of use to contemporary cases, while British or US designations should each be associated with their respective use cases).

\subsection{General observations : factors of brutality}

Before we start presenting a number of assorted examples, and the issues that each brings, which other researchers and programmers may face also, we start with a number of general observations about factors of brutality that stem from the work involved in putting this article together.

In reviewing and compiling the footage, a constant seems to be that most police brutality occurs at night, and in situations where large groups of police officers (e.g. 5 or more in the case of Rodney King, some active with some standing and watching, about 4 or 5 also in the case of Sterling Brown) face a single civilian; presumably, so, because, due to both of these factors, the officers involved are imbued with a sense of impunity. ${ }^{49}$

This sort of felt impunity most likely comes from the notion, real or false, of not being seen, or heard, and is of the kind that emerges out of group dynamics involving a majority and minority, where individual responsibility is thought to be diluted in the greater collective (guilt/responsibility).

But, in the case of Rodney King a nearby resident filmed from his balcony, who sent the outcome to a news station, while in the more recent case of Sterling Brown, police officers wore body cameras, footage of which was later released.

There is much reason to think that a systematic review of police brutality video recordings, the kind that on a large scale would only be possible with the establishment of a database, and practical only with the use of computers, would come to the conclusion that undue use of forces occurs in a ratio night : day that far outweighs daylight occurrences, and that large police groups : single officer ratios, as well as large police groups w/ 1 civilian : large groups w/ multiple civilians ratios, behave similarly.

However, factors of brutality should not be too different from general factors of violence. Primarily, asymmetry of power (in the broadest sense) $)^{50}$.

\footnotetext{
${ }^{49}$ The isolation of the victim seems to play a big role. The additional factor of the events happening at a time of reduced activity and visibility (e.g. late evening, nighttime) adds to the physical isolation that here often comes in the form of being encircled (Rodney King) or pushed back against some kind of end structure (e.g. a vehicle in the case of Sterling Brown).

${ }^{50} \mathrm{Be}$ it related to differences in force, physical strength, or professional or social status, or family hierarchies and dynamics, or specifically related to differences in age or sex or gender, or differences in economic statuses that make exploitation possible or unequal educational possibilities that have the same result. In societies where certain characteristics, religion, ethnic group, skin color, nationality, political affiliation, sexual orientation, gender identification and expression, are discriminating factors, these play a role as well.
} 


\subsection{Examples with related issues}

We start with a video that can be considered both ideal and realistic.

Ideal, because, as we discuss below, it is relatively easy to distinguish the various happenings. This can be attributed in part to the good (technological) quality of the recording ${ }^{51}$.

In other ways, the footage is realistic in the sense that it should be representative of most current recordings and recording abilities, and in the sense also that it presents a number of constraints (recorded at night, both civilian and police wear similar colors, presence of motion, etc.) that should represent some sort of worst case scenario in the analysis of such material.

In the arrest of Sterling Brown, a professional basketball player, the (bodycamera) footage gives multiple clues as to the presence of (many) police officers, and one civilian : badges on the torso, insignia on the lower arms, the large inscription saying "POLICE" on the back, and the dark blue uniforms, or/and the absence of such. ${ }^{52}$

Remarkable here is the rapid escalation - 6 seconds - from one state, which can only be described as uneventful, to another driven by one of the police officers and resulting in an escalation of force, and ultimately the use of a stun gun for a situation that started as a simple "Can I see your driver's license?"

\footnotetext{
51 "1080p" : $1920 \times 1080$ pixels, corresponding to width and height of the image. Average bitrate was approx. 1500 kilobits per second (kbps), video compression standard H.264.

${ }^{52}$ The images were extracted from footage published by the New York Times : https: //www . youtube . com/watch?v=qXbgTQfMNm4.
} 


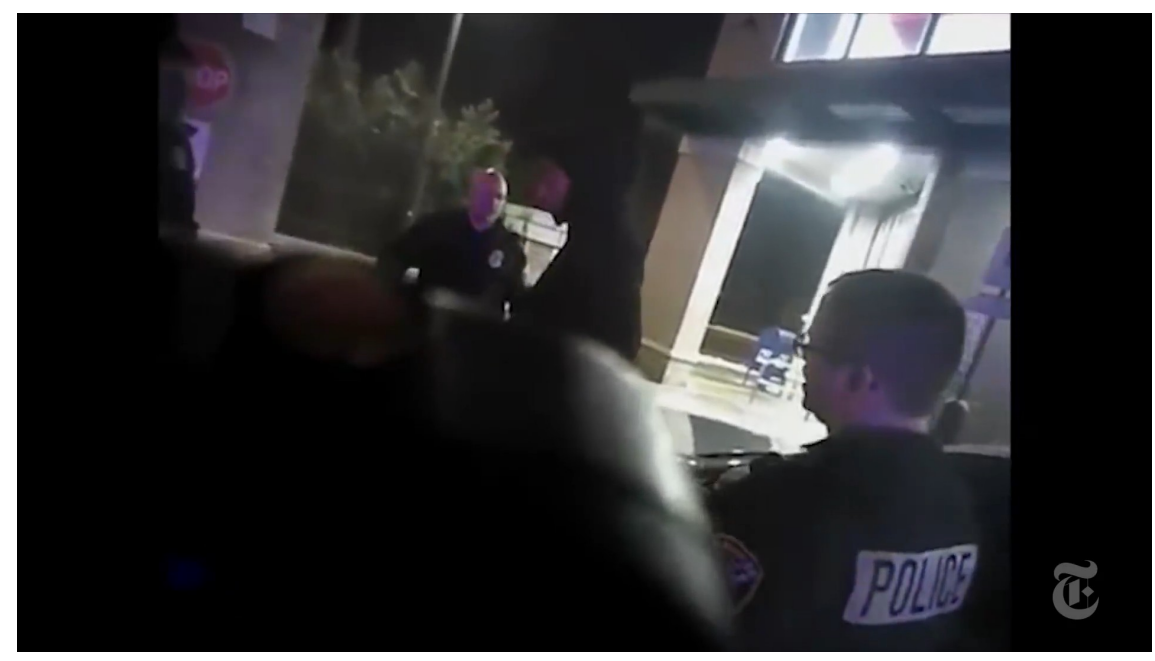

(a) $03 \mathrm{~m}: 08 \mathrm{~s}$

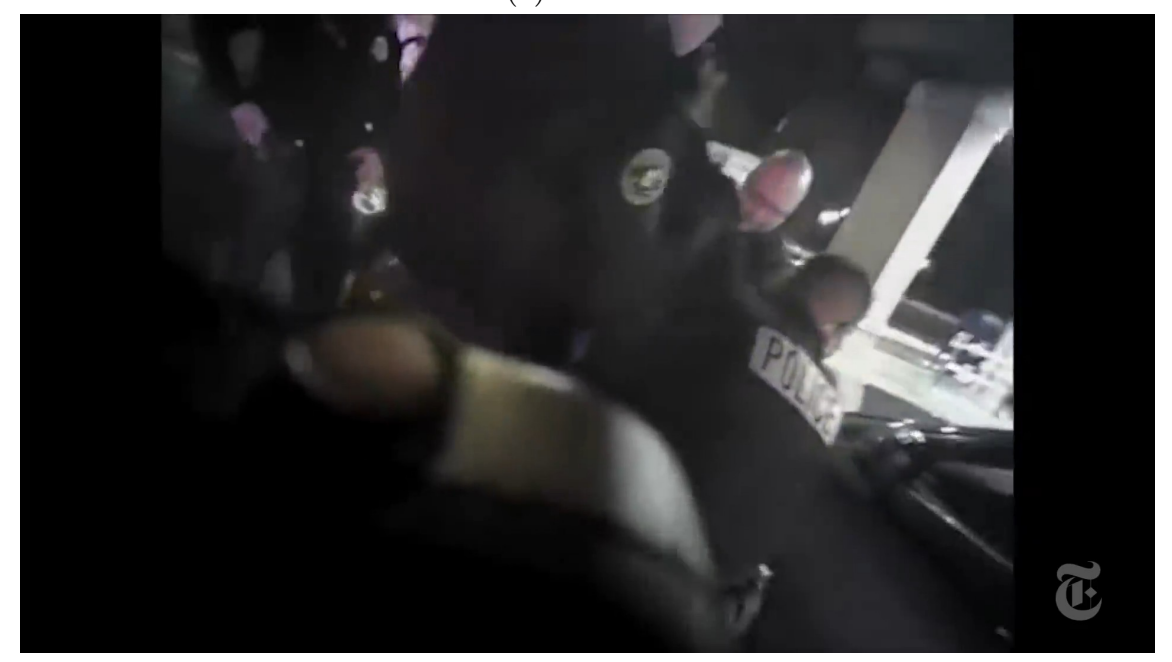

(b) $03 \mathrm{~m}: 14 \mathrm{~s}$

Figure 1: The stun gun arrest of Sterling Brown (2018)

But, such detection is not always so easy, and such signs are not always so readily given away, as the next two examples show or demonstrate.

In one of the most notable examples of police brutality in newer history, the case of Rodney King, difficulties from correctly matching the footage result from the fact that, despite similar adverse conditions, the poor quality of the footage leads to all sorts of obstacles that amplify the fact that the events not only occurred at night (as in the case of Sterling Brown), but that they were filmed from a distance, presumably making the use of a (it is presumed digital) zoom necessary, further deteriorating the quality of footage and further accelerating 
the deterioration of colors (it is hard to tell whether the officers were wearing dark blue or black, or indeed blue rendered black).

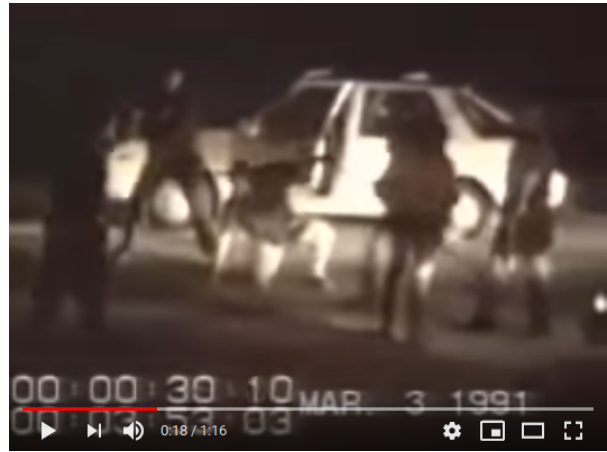

(a) -

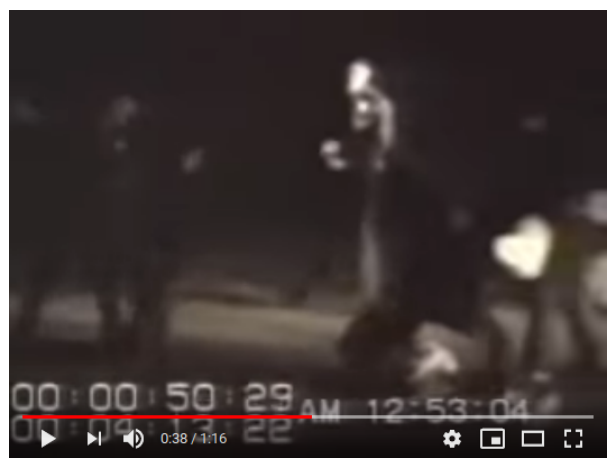

(c) 20 seconds later

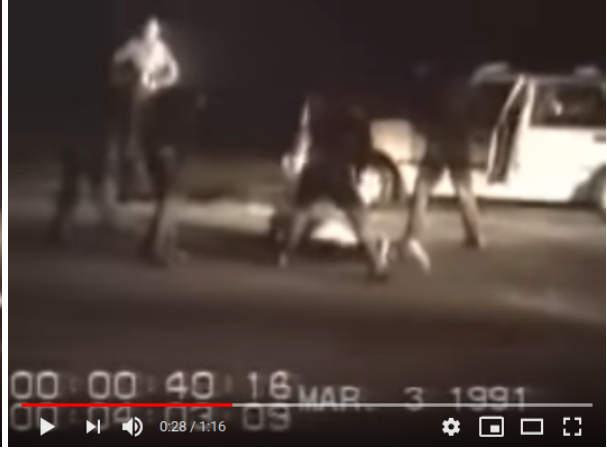

(b) 10 seconds later

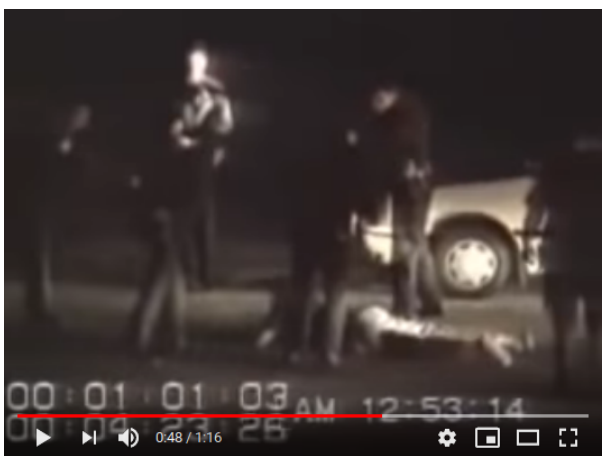

(d) 30 seconds later

Figure 2: The beating of Rodney King (1991)

In, this time, a fictional example, whose realism should be ascertained, the 1981 film by Sidney Lumet runs for two hours, but rarely if ever shows police officers in any homogeneous or recognizable uniforms (bar badges). This would further complicate correct detection by the algorithms discussed here, and we present two examples of this below. 


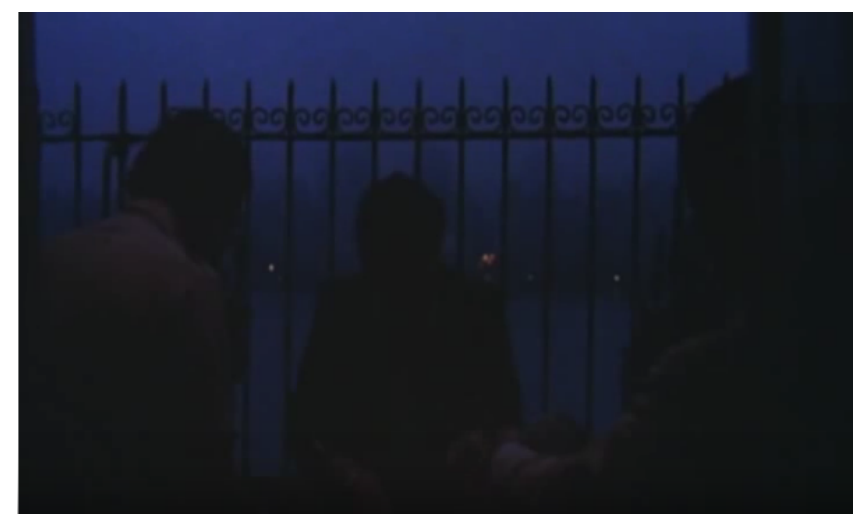

(a) at night

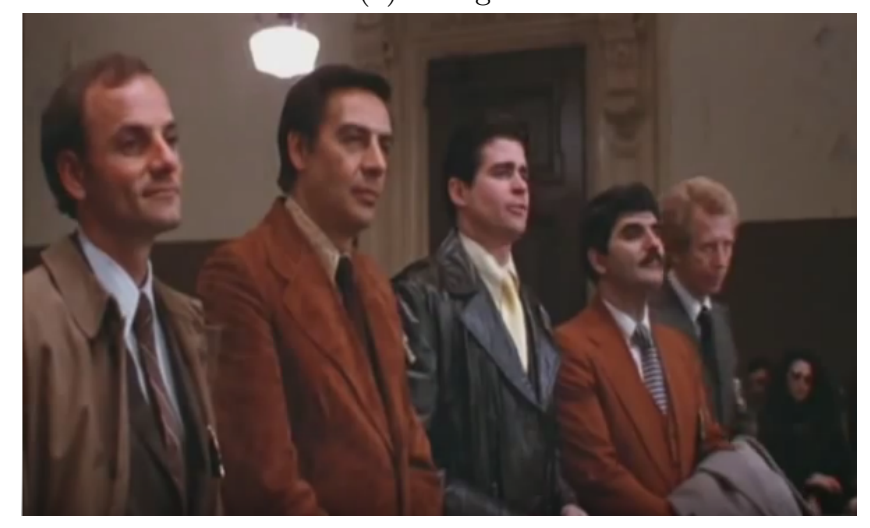

(b) at day

Figure 3: Prince of the City (1981)

The designers of such a program will consider that other professions require uniforms are worn and whose appearance may falsify the results of the objects (e.g. plumber, mailman, etc.), possibly in both ways.

Finally, tests would include aberrant footage, such as Blue, a film by Derek Jarman, where only the color blue is shown ${ }^{53}$.

\footnotetext{
${ }^{53}$ Affected with AIDS, and its side-effects, he had become particularly sensitive to that color.
} 


\section{BLUE}

(a) Credits.

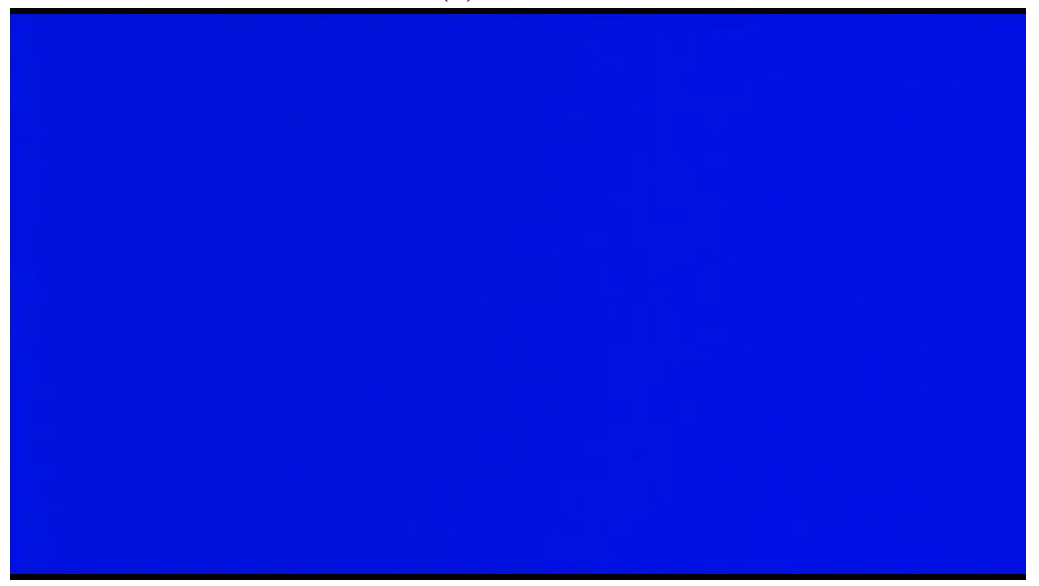

(b) Film.

Figure 4: Blue (1993)

Other thinkable edge cases or outliers can be constituted by a yellow lego placed on blue lego background, and similar such constructs.

But, if algorithms such as the kind presented in Karpathy et al. can accurately detect mountain biking from other, partly very niche but related activities like "mountain unicycling", there is no reason to believe they would not be able do the same between "police" and "mailman" or delivery personnel.

\subsection{Presentation of results}

The results of running such a program on a set of footage could be uploaded to an openly accessible platform specializing in video content, and additionally self- 
hosted to prevent any censorship or otherwise motivated takedown of content. ${ }^{54}$

\section{Description (of the program, functionalities)}

To summarize :

1. Such a program needs training data, in other words a dataset (database).

The steps of which involve collection and labeling (which should not necessarily be seen as separate).

NB. neither of these operations represents any particular technical challenge and could and should be organized as a group or community effort.

2. Such a program would be modeled after Karpathy et al. 2014. (Or, any research that has superseded them.)

This has the advantage that their method has already proven to be successful (based on their dataset and based on results they have selected and provide).

3. (Because of 2.) Such a program would use convolutional neural networks (Abbr. : CNN, convnets, convolutional networks, ...).

4. (Because of 2.) Such a program would use downpour stochastic gradient descent (Dean et al. 2012), or any newer optimization algorithm (Ruder 2016).

5. Such a program would make use of an appropriate (e.g. machine learning or deep learning) library, whose in-built functions are likely to have implemented 3. and 4. and many related operations or otherwise needed functionalities.

6. Choice of architecture : each architecture leads to a different program. There is no single program such as the one described here (just as there is no one Minerva). (This would possibly also affect the design of the dataset.)

A brief and overly simplified description of such a program's architecture would look like so :

Input : video (i.e. many successive images).

Output : an adequate description of the object displayed, in the form of a string (e.g. "Police"), paired with the probability of a correct match (e.g. "70\%" or "0.7"); possibly, an "information" 55 .

\footnotetext{
${ }^{54}$ See Karpathy et al.'s own approach to this, https://cs . stanford.edu/people/karpathy/ deepvideo/ and elsewhere.

${ }^{55}$ Such an information would be triggered based on the estimated probability of a match, the threshold of which can be freely determined. In more intricate implementations, a signal, once passed the threshold, would vary in intensity or rhythm (e.g. ., . ., . . ., ...) depending on levels of probable matches. Otherwise, modulation of the signal could occur according to the amount of identified matching objects (e.g. $>5,>10,>50$ ). Modulation could also occur according to relevant variables such as day/night.
} 
In the event of a non-match such a program would simply render a value of e.g. "non-match" or "non-Police", and the probability (which would be greater than the probability of a match) associated with it.

\section{$5 \quad$ Legal aspects}

Due to the unusual nature of this program, unusual though only in the sense that it would exist inside a system whose dominant values, of which Marx wrote that at any time they were simply the values of the dominant class, are antagonistic to it, we feel the need to address some of the legal issues that would surround the making of such a program.

\subsection{Programs as science and research}

Such a program could be conceived as a research project, a "proof of concept", a prototype, submitted as a journal article, published as open access literature, submitted as a thesis.

The makers of such a program would then likely benefit from the same rights and protections usually awarded to these use cases.

Though, there is no guarantees for this. Institutions such as MIT for example have in multiple instances, so in the case of Andrew Huang, turned their backs on students - to say nothing of the case of Aaron Swartz. This appears to be in stark contrast with earlier time periods and eras whose exploits these same schools now owe their current, and temporary, reputation to (a transformation no doubt codependent with the changing characteristics of these institutions' administrations and new type of administrators, recruited from business, finance and legal backgrounds, as opposed to the more strictly academic or scholarly origins of their predecessors).

The exact legal situation, and the amount and extent of these rights, is likely to be different from country to country, and possibly state to state where countries have adopted a federal system. It is further likely to differ from university to university, particularly in countries whose educational system is structured in such a way that private and public institutions co-exist, with the former presumably less interested in the welfare of a single individual if on the other hand financial interests were at stake or legal threats surfaced (public, or state, institutions would likely be more constrained by various applicable laws).

\subsection{Programs as art and "protest programs"}

A program such as the one described here could be conceived as art.

The makers of such a program would then benefit from the same rights and protections usually awarded to artists. ${ }^{56}$

\footnotetext{
${ }^{56}$ This is also not infallible. See, for example, the trial of Baudelaire surrounding Les Fleurs $d u$ mal (he was fined, along with his editors, but received no prison sentence, portions of his book of poems were censored but it went back on sale, and his fame only accrued afterwards).
} 
In such an event, the status of programming as an "art" would, and possibly would need to be discussed and a successful presentation would in part most likely depend on the rendering of a series of authoritative references (e.g. "The Art of Computer Programming").

Specifically, such a program could be conceived, in the manner of political art, as a "protest program", which it would be. Refer, for example, to protest songs, and protest art, and the case law.

\section{The system's sociology}

In explaining the origins of the programming language Smalltalk, Adele Goldberg noted that much of it could be traced back to what she called the "system's sociology". We will end by answering this question as well.

One obstacle to such a program - beyond the obvious sociological reasons that make it so that such an endeavor is unlikely to be the priority of prime time computer science journal publications, or computer scientists, and that beyond that, such and similar programs are not made by outsiders who are less likely to care for or be hindered by the aforementioned considerations - is undoubtedly the specialized field literature.

However, it is arguable whether or not knowledge of this literature is necessary for the development of the outlined program.

Whereas in previous generations of artificial intelligence researchers, not only a knowledge of the mathematics underlying the algorithms was necessary, but the implementations themselves often required much technical prowess considering the platforms they were to run on and the languages they were written in, the combination of which resulted in very small, elite communities, this cannot be said to be true anymore.

This is the case now more than ever that a variety of libraries exist that take care of much of the underlying work by providing the user with a collection of pre-made, ready-to-be-used functions which cover most of the phases involved in such a work (training on data, etc.).

\section{Conclusion}

Every age brings with itself its needs of new terminologies and philosophies to help make sense of its own specific changes, which are rarely those of previous

ages : the concerns of the philosophers of the Industrial age were not those of the scholars of Christianity, Dante, Aquinas or St Jerome, just like theirs did not correspond to those of the Ancient Greeks, uninterested as they were and could only be in any issues relating to the monotheistic religions of their successors. Ours are not theirs.

But, their experiences, and their writings help us : in this article, a prototype program named "Minerva and Virgil" (Miv, MinVir or Maeve) was presented whose outline described a tool that is thought to be useful to those who are 
exposed to State brutality - a term we have proposed as the generalized form of the already accepted "police brutality". Examples of which we have given, and examples of which we are given on an almost daily schedule. To those who say, perhaps hoping for no answer "But, what solution?" : one is contained here, in one form or another.

Disappointments in this paper will no doubt stem from the fact, that, in a tradition perfected in mathematics textbooks, the problems are left as an exercise to the reader.

While some other readers wished for more of the equations they are accustomed to from other papers, to convince themselves of the overall goodness of it all, but mostly to ascertain the true nature of such a publication as a proper computer science publication; an act by which they filter out in fact those unlike themselves. Their thoughts and aways : a Christian like Dante to an Ancient Roman like Virgil, so foreign as the Menrva of the Etruscans compared to the Minerva of later civilizations. To make sure that they can stay amongst themselves, so that they can stay with the others as long as due, and that them are never us too.

But, if today's computer scientists, who are likely to be very different from those we will hopefully know tomorrow, were not so busy competing for a chance to become a cog in the wheel of one or the other of the currently large companies, to apply there, their immense smarts, but general lack of wisdom, and lack in any case of either much common sense or moral concerns, computer science journals would be filled with such and similar problems, and their solutions. And, they would be praised, and seen as what they are : the rare combination of, it appears, mostly mutually exclusive traits, technical skills and social courage. Alas, these gentlemen would rather work on chatbots. As Virgil with Dante, we can only go so far, and note "In this circle are those who have made reason slave to appetites". 


\section{Bibliography}

Bishop, Christopher. 2006. Pattern Recognition and Machine Learning. New York, NY : Springer.

Chollet, Francois. 2017. Deep Learning with Python. Shelter Island, NY : Manning.

Dean et al. 2012. "Large Scale Distributed Deep Networks". In : Advances in Neural Information Processing Systems 25. http://papers.nips.cc/paper/ 4687-large-scale-distributed-deep-networks.pdf

Geron, Aurelien. 2017. Hands-On Machine Learning with Scikit-Learn $\&$ TensorFlow. Sebastopol, CA : O'Reilly.

Goodfellow et al. 2016. Deep Learning. Cambridge, MA : The MIT Press.

Hastie et al. 2017. The Elements of Statistical Learning. https://web. stanford.edu/ hastie/ElemStatLearn/printings/ESLII_print12.pdf Additional resources :

https://web.stanford.edu/ hastie/ElemStatLearn/

Karpathy et al. 2014. "Large-scale Video Classification with Convolutional Neural Networks". https://cs.stanford.edu/people/karpathy/ deepvideo/deepvideo_cvpr2014.pdf

Additional resources : https://cs.stanford.edu/people/karpathy/deepvideo/

Le Cun et al. 1989. "Handwritten digit recognition with a back-propagation network". In : Advances in Neural Information Processing Systems 2. https://papers.nips.cc/paper/ 293-handwritten-digit-recognition-with-a-back-propagation-network. pdf

Additional resources :

http://yann.lecun.com/exdb/lenet/

Lecun et al. 1998. "Gradient-Based Learning Applied to Document Recognition" Proceedings of the IEEE 86(11) : 2278-2324.

http://yann.lecun.com/exdb/publis/pdf/lecun-98.pdf

Ruder, Sebastian. 2016. "An overview of gradient descent optimization algorithms". ruder.io/optimizing-gradient-descent/

SethBling. 2015. "MarI/O - Machine Learning for Video Games". https://www . youtube. com/watch?v=qv6UVOQ0F44

—. 2015b. "MarI/O Followup: Super Mario Bros, Donut Plains 4, and Yoshi's Island 1". https ://www . youtube. com/watch?v=qv6UVOQOF44

—. 2017. "MariFlow - Self-Driving Mario Kart w/Recurrent Neural Network". https : //www . youtube . com/watch?v=qv6UVOQOF44 
Stanford. 2017. "Lecture Collection — Convolutional Neural Networks for Visual Recognition (Spring 2017)". https://www . youtube.com/playlist? list=PL3FW7Lu3i5JvHM81jYj-zLfQRF3E08sYv

Stanley, Kenneth, and Miikkulainen, Risto. 2002. "Evolving Neural Networks through Augmenting Topologies".

http://nn.cs.utexas.edu/downloads/papers/stanley.ec02.pdf

Additional resources :

http://nn.cs.utexas.edu/?stanley:ec02 